\title{
Ab initio Calculations of Absorption Coefficients of Small Carbon Hydride Molecules
}

\author{
S.D. Peyerimhoff
}

Institut für Physikalische und Theoretische Chemie, Universität Bonn, Wegelerstraße 12, 53115 Bonn, Germany

\section{Introduction}

Quantum chemical methods have become a powerful tool for investigating the electronic structure of small molecules in ground and excited states. This is particularly true for species which are not easily accessible by experiment, either because they are short-lived, for example, or unstable under laboratory conditions or difficult to detect since their signals are hidden by other, prevailing molecules. In this sense theoretical methods are of special importance for molecules in atmospheric, stellar and interstellar environments.

The present contribution will focus on the use of ab initio computations to determine the absorption of light by molecules and will exemplify some aspects on the spectra of $\mathrm{CH}_{2}^{+}, \mathrm{C}_{2} \mathrm{H}$ and their deuterated species.

\section{General considerations on the ab initio calculation of radiative transitions in molecules}

Calculations for molecules are generally based on the Born-Oppenheimer approximation, i.e. the separability of nuclear and electronic motion is assumed and the coupling terms between the two types of motion are neglected. The total wavefunction is then a product of an electronic part $\phi_{e}(\mathbf{r}, Q)$ and a function $\chi(\mathbf{Q})$ describing the nuclear motion; the latter depends on the nuclear coordinates $\mathbf{Q}$, the former on the electronic coordinates $\mathbf{r}$ and is evaluated at a fixed nuclear arrangement, i.e. it depends only parametrically on the nuclear position $Q$. Neglect of the coupling terms $\partial \phi_{e} / \partial \mathbf{Q}$ and $\partial^{2} \phi_{e} / \partial \mathbf{Q}^{2}$ (which arise from the nuclear kinetic energy operator) is justified if the electronic wavefunction does not change significantly with the nuclear coordinates; if $\phi_{e}$ changes considerably along a nuclear coordinate the validity of the Born Oppenheimer approximation is questionable.

In order to obtain the potential energy surface $U(\mathbf{Q})$ one has to solve the electronic Schrödinger equation

$$
\hat{H}_{e} \phi_{e}=E_{e} \phi_{e}
$$


for a number of nuclear positions $Q$ and then obtain $U(Q)=E_{e}(Q)+V_{N N}$ point by point, where $V_{N N}$ is the nuclear repulsion energy. In order to obtain vibrational levels, it is necessary to solve the nuclear Schrödinger equation

$$
\left(\hat{T}_{N}+U\right) \chi_{\nu}=E \chi_{\nu}
$$

where $\hat{T}_{N}$ is the vibrational kinetic energy operator and are $\chi_{\nu}$, the vibrational wavefunctions. More details of potential energy calculations on ground and excited states can be found elsewhere (Bruna \& Peyerimhoff 1987).

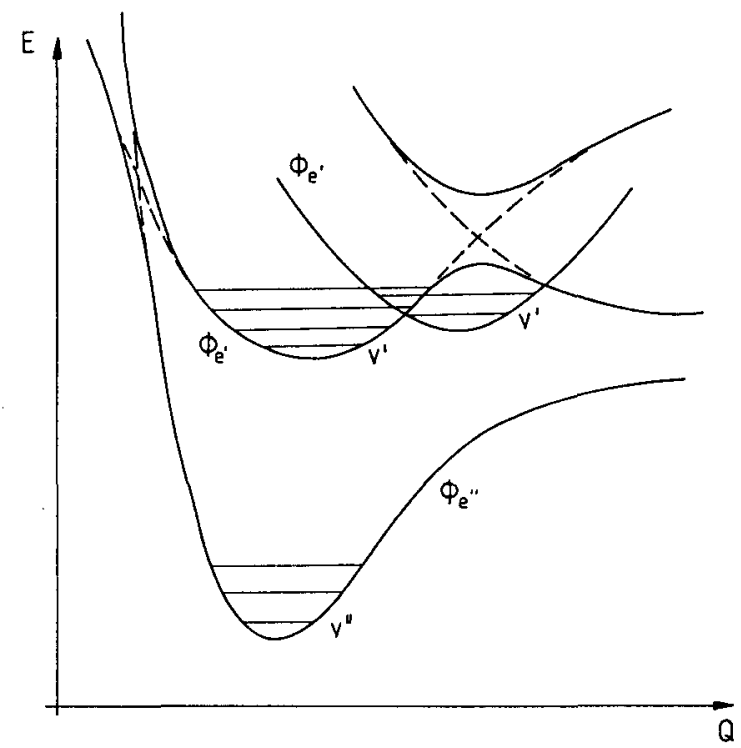

Fig. 1. Typical examples for potential curve interactions: dotted lines refer to diabatic states of the same symmetry, their adiabatic counterparts show avoided crossings.

A typical example of potential energy surfaces is presented in Fig. 1. If transitions occur between two separate curves, the oscillator strength as a measure of the absorption strength is simply evaluated as

$$
\begin{aligned}
f_{e^{\prime \prime} e^{\prime} \nu^{\prime \prime} \nu^{\prime}} & =\frac{2}{3} \mid\left\langle\phi_{e^{\prime \prime}} \chi_{\nu^{\prime \prime}}|\boldsymbol{\mu}| \phi_{e^{\prime}} \chi_{\nu^{\prime}}>\left.\right|^{2} \Delta E_{e^{\prime \prime} e^{\prime} \nu^{\prime \prime} \nu^{\prime}}\right. \\
& \equiv \frac{2}{3}\left|<\chi_{\nu^{\prime \prime}}\right| R_{e^{\prime} e^{\prime \prime}}(Q)\left|\chi_{\nu^{\prime}}>\right|^{2} \Delta E_{e^{\prime \prime} e^{\prime} \nu^{\prime \prime} \nu^{\prime}}
\end{aligned}
$$

In this formula quantities with double and single prime refer to the ground and the electronically excited state, respectively. The quantities $\phi_{e}$ and $\chi_{\nu}$ are obtained from Eqs. 1 and $2 ; \boldsymbol{\mu}$ is the dipole operator and integration is over $\mathbf{r}$ and $\mathbf{Q}$. The quantity $R_{e^{\prime} e^{\prime \prime}}$ is the electronic transition moment. The relations between oscillator strength, lifetime and other quantities measuring the absorption strength are well documented (Herzberg 1950, Peyerimhoff 1992, Larsson this volume). 
Frequently Eq. 3 is simplified by taking the average of $R_{e^{\prime} e^{\prime \prime}}$, assuming it is independent of the nuclear coordinate (Franck-Condon principle)

$$
f_{e^{\prime \prime} e^{\prime} \nu^{\prime \prime} \nu^{\prime}}=\frac{2}{3}\left|R_{e^{\prime} e^{\prime \prime}}\right|^{2}\left|<\chi_{\nu^{\prime \prime}}\right| \chi_{\nu^{\prime}}>\left.\right|^{2}
$$

Hence, very often only the electronic part $R_{e^{\prime} e^{\prime \prime}}$ and the vibrational overlap $\left\langle\chi_{\nu^{\prime \prime}} \mid \chi_{\nu^{\prime}}\right\rangle$ are considered when discussing absorption strengths.

Numerous calculations of absorption strengths according to Eq. 4 are in the quantum chemistry literature. Generally, errors are of the order of $10 \%$ or less if suitable treatment is chosen to obtain the electronic wavefunctions. In many instances computed values are the only ones available when measurements of absolute absorption strength have yet to be carried out.

A more extended treatment is required if potential surface interaction occurs, a situation which becomes increasingly important for higher excited states and poly atomic molecules with several degrees of freedom. Frequent coupling mechanisms are the coupling due to the nuclear kinetic energy operator ("breakdown" of the Born-Oppenheimer approximations, as mentioned above) which generally couples states of the same symmetry and the spin-orbit and Renner-Teller interactions coupling states of different symmetry. Furthermore, the zero-field splitting of the spin multiplets of a single state may become as large as the energy separation between different states in molecules containing heavy atoms (large spin-orbit effects) and must also be taken into account for the computation of the absorption strength. In such cases the simple quantity of Eq. 4 serves as a guideline for the magnitude of the absorbing power but may deviate considerably from reality.

A typical example for the interaction of many potential surfaces of the same symmetry is given in Fig. 2 which shows the bending curves for excited states in $\mathrm{HCO}$. Two valence states (denoted by V), one with its minimum at linear geometry and the other preferring a bent $\mathrm{HCO}$ molecule with an angle around $90^{\circ}$, couple with the series of Rydberg states of ns, np $\pi$ and np $\sigma$ symmetry, all of which prefer the linear $\mathrm{HCO}$ arrangement; their bending curves run fairly parallel to the Rydberg state limit, i.e. the $\mathrm{HCO}^{+}$ion. The perturbed states (solid curves in Fig. 2) show a complicated pattern for the bending potential curves with various shoulders, barriers and wells which can be rationalized based on the dotted unperturbed (diabatic) curves. Note that only the Rydberg states up to $n=4$ are considered in the figure and that the nd series has not explicitly been taken into account. The first excited state, for example, is characterized as a valence state in the region between $180^{\circ}$ and $160^{\circ}$, has Rydberg character for bending angles between $160^{\circ}$ and $100^{\circ}$ and regains more compact valence character for smaller bond angles. The intensity of a transition from the ground state to this first excited state thus changes drastically with the angular coordinate (the intensity is generally weak for Rydberg-like transitions and stronger for suitably allowed valence-like transitions); a very irregular spectrum in the entire wavelength region is the consequence of such interactions. Such couplings occur not only in the bending but also in the stretching coordinate as discussed by Lorenzen-Schmidt et al. (1993) and Perić \& Peyerimhoff (1993a,b). 


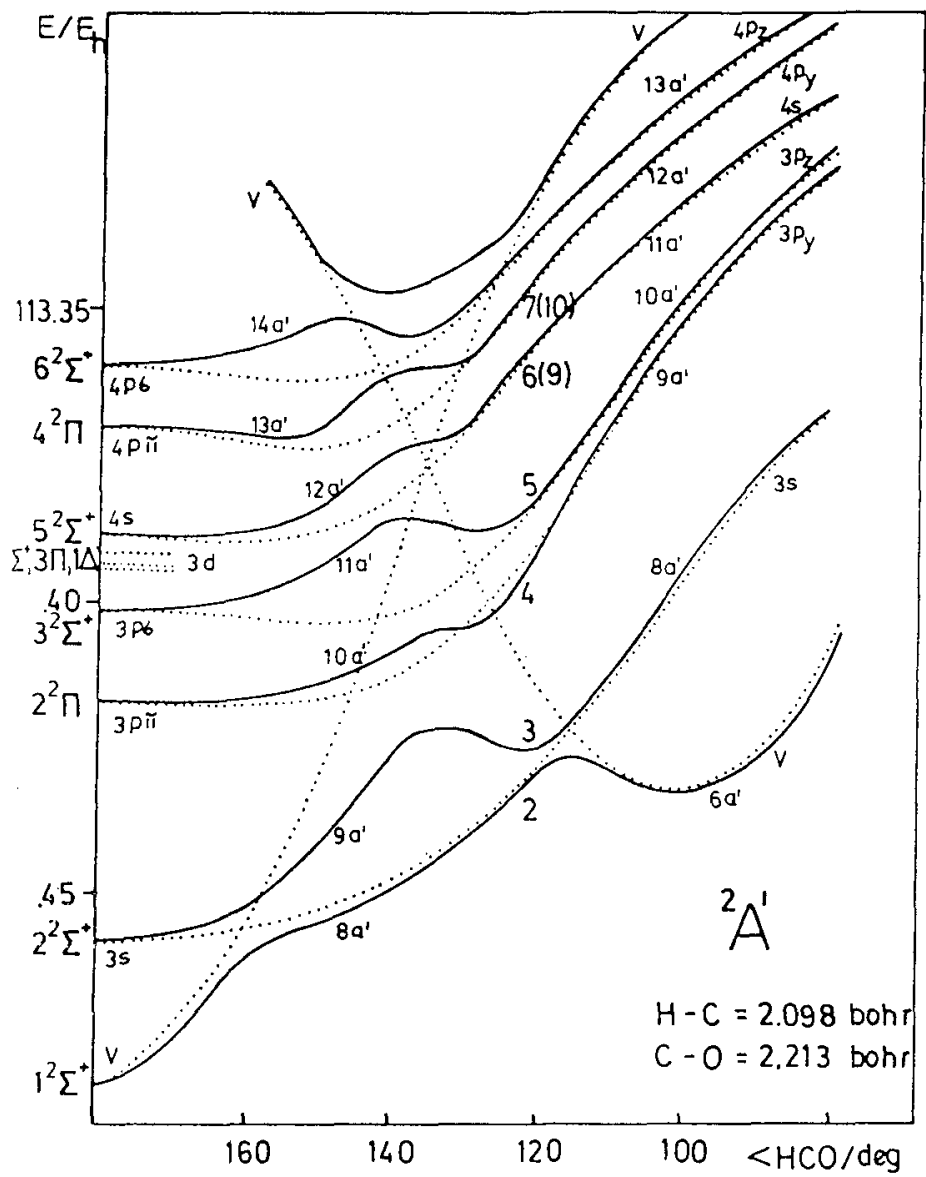

Fig. 2. Calculated potential curves for the excited states of $\mathrm{HCO}$ as a function of the bending angle $\left(180^{\circ} \equiv\right.$ linear $)$. The dominant molecular orbital in a given state is indicated. Dotted lines refer to the diabatic representation (same character) of states.

Such "non-adiabatic" interactions are often also the cause for non-radiative deactivation. In rare gas hydrides such as $\mathrm{ArH}$ and $\mathrm{HeH}$, for example, interactions between the repulsive inner part of the potential curves as indicated in Fig. 1 lead to much faster deactivation of the excited states to the ground state than do the radiative transitions (Hemert et al. 1986, Hemert \& Peyerimhoff 1991); this mechanism explains why the search for the corresponding emission spectrum was unsuccessful.

A representative example for the coupling due to spin-orbit interaction is the interaction of the $\mathrm{X}^{1} \Sigma^{+}$and $\mathrm{a}^{3} \Pi$ states in $\mathrm{MgO}$ which is isovalent to the $\mathrm{C}_{2}$ molecule. The situation is shown schematically in Fig. 3. The strength of the vibronic coupling depends on: (a) the magnitude of the spin-orbit coupling between ${ }^{1} \Sigma^{+}$and ${ }^{3} \Pi$, (b) on the form of the unperturbed vibrational wavefunctions in 


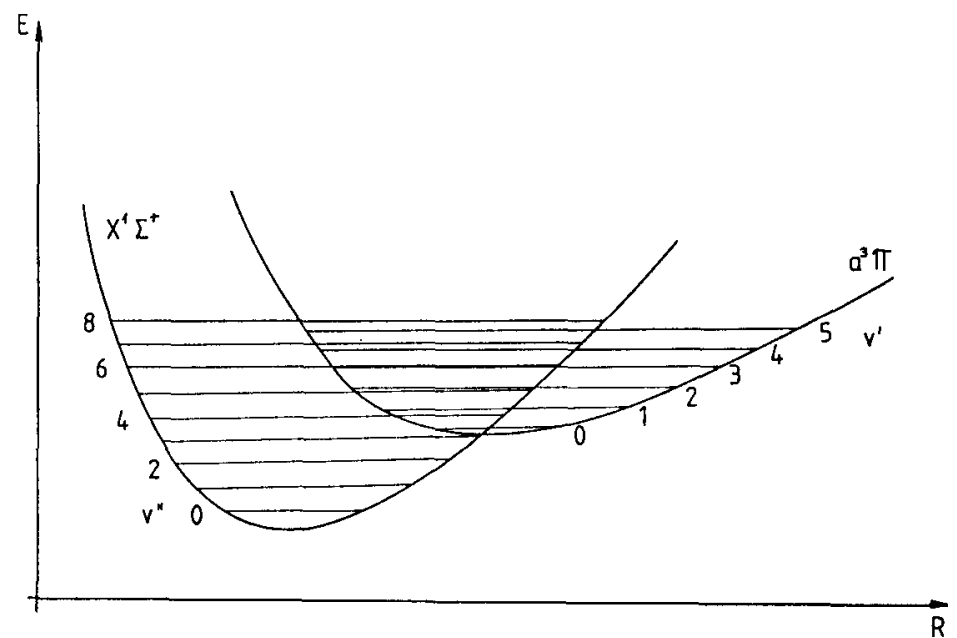

Fig. 3. Schematic representation of the interaction of the $\mathrm{X}^{1} \Sigma^{+}$and $\mathrm{a}^{3} \Pi$ states in $\mathrm{MgO}$.

the ${ }^{1} \Sigma^{+}$and ${ }^{3} \Pi$ potential wells, in particular their possible spatial overlap and (c) on the relative energy of the vibrational levels in the two potential wells.

Fig. 4 shows the computed vibronic functions. The ${ }^{3} \Pi(v=0,1)$ and $\mathrm{X}^{1} \Sigma^{+}(v=$ $3,4)$ are essentially unperturbed (Thümmel et al. 1989). The ${ }^{3} \Pi(v=2)$ function already shows admixture of the ${ }^{1} \Sigma^{+}(v=5)$ function in the area of the inner turning point; ${ }^{3} \Pi(v=3)$ and ${ }^{1} \Sigma^{+}(v=6)$ are in near energetic resonance and hence the two vibronically mixed functions have very different character as compared to their unperturbed counterpart. For higher vibrational levels the degree of mixing becomes small again. Absorbtion characteristics for "infrared" transitions (within the ${ }^{1} \Sigma^{+}$and the ${ }^{3} \Pi$ vibrational states) or "electronic" transitions (between ${ }^{1} \Sigma^{+}$and ${ }^{3} \Pi$ ) are thus markedly changed as soon as the strongly perturbed levels are involved. Ab initio treatments can fairly easily compute the necessary spinorbit matrix element, the unperturbed vibrational functions and the actual coupling. However, their accuracy is generally not high enough to determine the relative location of the interacting levels to within a few wavenumbers so that information about the relative location of potential surfaces to this accuracy has to come from other sources (spectroscopy).

Vibronic coupling is not very effective if the overlap between the unperturbed vibrational wavefunctions of the two coupling states is small, even if near energy resonance of levels and strong coupling is present. This has been shown by Gemein \& Peyerimhoff (1991) for the $\mathrm{X}^{1} \Sigma^{+}$and $\mathrm{a}^{3} \Pi$ and $\mathrm{a}^{3} \Sigma^{+}$states in CO; the ground state vibrational levels which could possibly mix possess quantum numbers $v>30$ and therefore have so many "wiggles" that the overlap integral with the ${ }^{3} \Pi$ or ${ }^{3} \Sigma^{+}$wavefunctions having small vibrational quantum numbers is very small. This observation that vibronic coupling is not very efficient if the difference in the vibrational quantum numbers of the unperturbed states is very large can be considered as a general rule. 


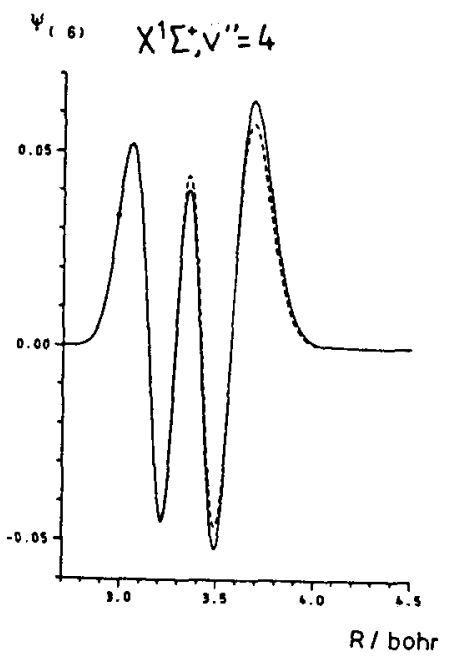

$$
\psi, n \quad a^{3} \Pi_{\sigma^{\prime}}, v^{\prime}=1
$$
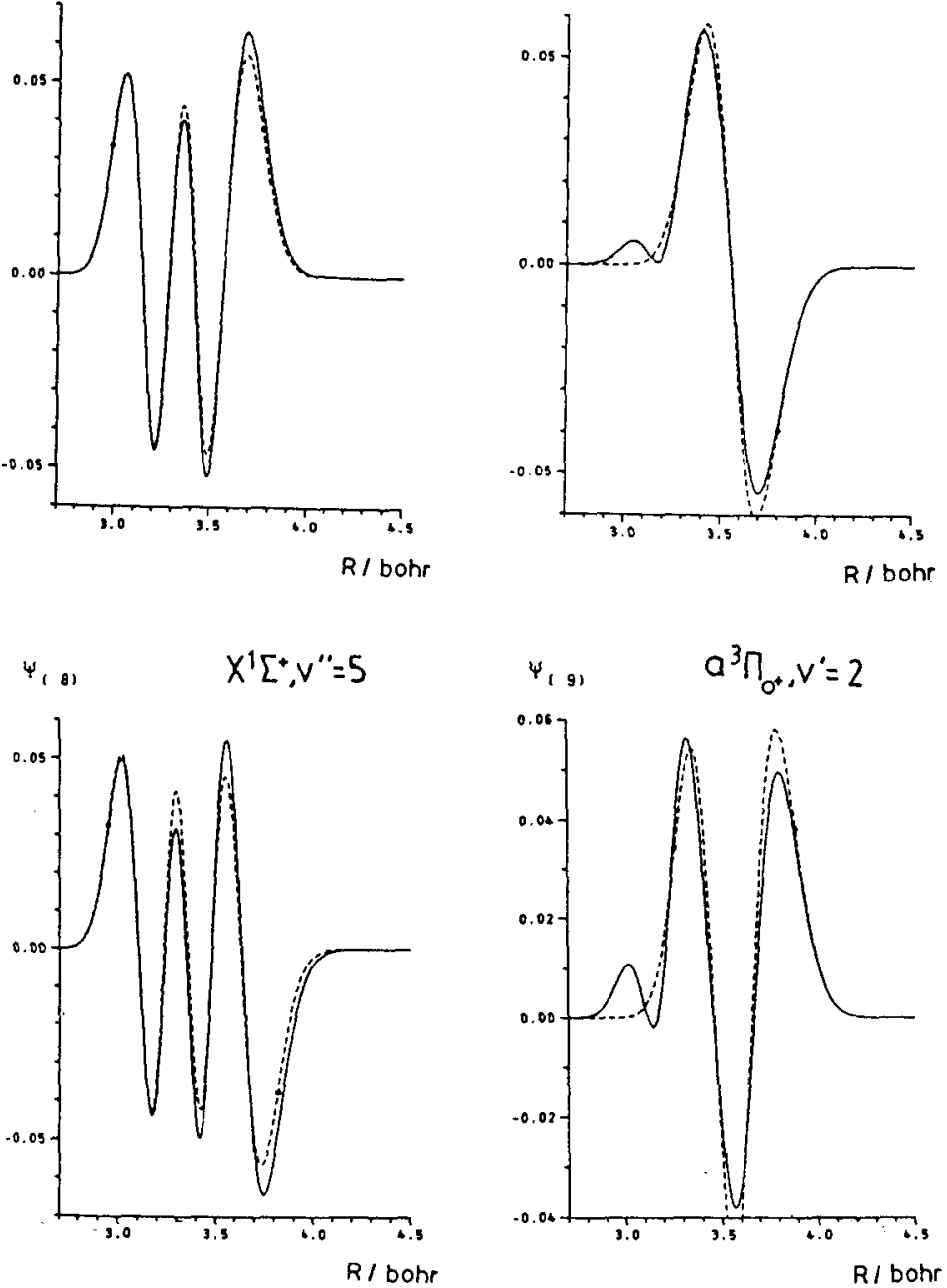

Fig. 4. Calculated vibronic wavefunctions in the $\mathrm{X}^{1} \Sigma^{+}-a^{3} \Pi$ interaction zone of $\mathrm{MgO}$. For labelling of states see Fig. 3. The dotted lines refer to the unperturbed vibrational functions.

Finally, an example for the importance of zero-field splitting can be found in the work of Marian (1990) on $\mathrm{NiH}$, which is also briefly discussed by Peyerimhoff (1992). The NiH ground state ${ }^{2} \Delta$ is followed by ${ }^{2} \Sigma^{+}$and ${ }^{2} \Pi$ states. Spin-orbit interaction $(L+S)$ mixes components of ${ }^{2} \Delta$ and ${ }^{2} \Pi$, as well as of ${ }^{2} \Pi$ and ${ }^{2} \Sigma^{+}$, so that the correct potential curves are ${ }^{2} \Delta_{5 / 2},{ }^{2} \Delta_{3 / 2},{ }^{2} \Pi_{3 / 2},{ }^{2} \Pi_{1 / 2}$ and ${ }^{2} \Sigma_{1 / 2}^{+}$. The vibronic functions then also have character of the different electronic states. 


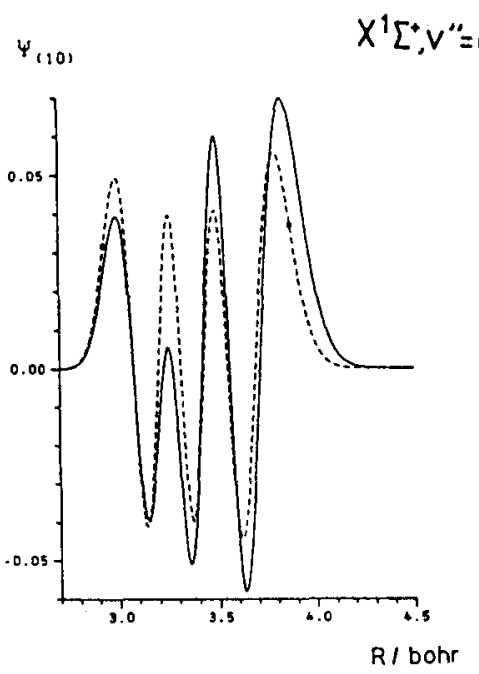

$\psi_{111} a^{3} \Pi_{0}, v^{\prime}=3$
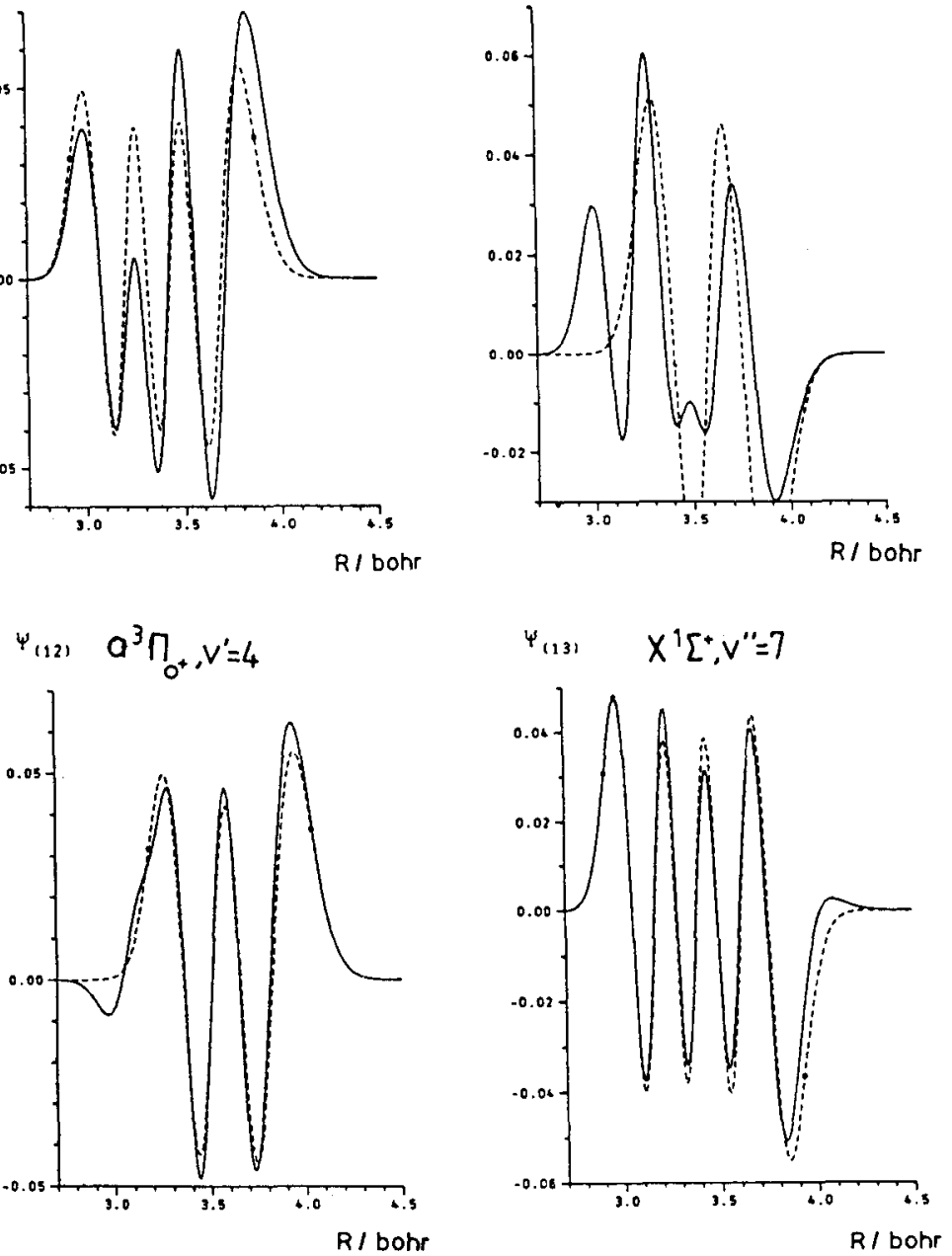

Fig. 4. - continued. Calculated vibronic wavefunctions in the $\mathrm{X}^{1} \Sigma^{+}-a^{3} I I$ interaction zone of $\mathrm{MgO}$. For labelling of states see Fig. 3 . The dotted lines refer to the unperturbed vibrational functions.

\section{The spectrum of $\mathrm{CH}_{2}^{+}$}

The occurrence of the $\mathrm{CH}_{2}^{+}$ion in mass spectroscopy is well known since its detection (Hogness \& Kvalness 1928) 65 years ago; this ion is furthermore assumed to be an important partner in chemical reactions. The first spectroscopic indication for its existence came in 1961 from Herzberg (1961) and since then various efforts to characterize this ion spectroscopically have failed to be success- 
ful. Only very recently (Rösslein et al. 1992) were the infrared transitions of the $\nu_{3}$ band seen. The best characterization of this molecule comes from various ab initio calculations (Bartholomae et al. 1981, Reuter \& Peyerimhoff 1992). The bending potential curves for the two lowest states are seen in Fig. 5 as taken from our own work (Reuter \& Peyerimhoff 1992).

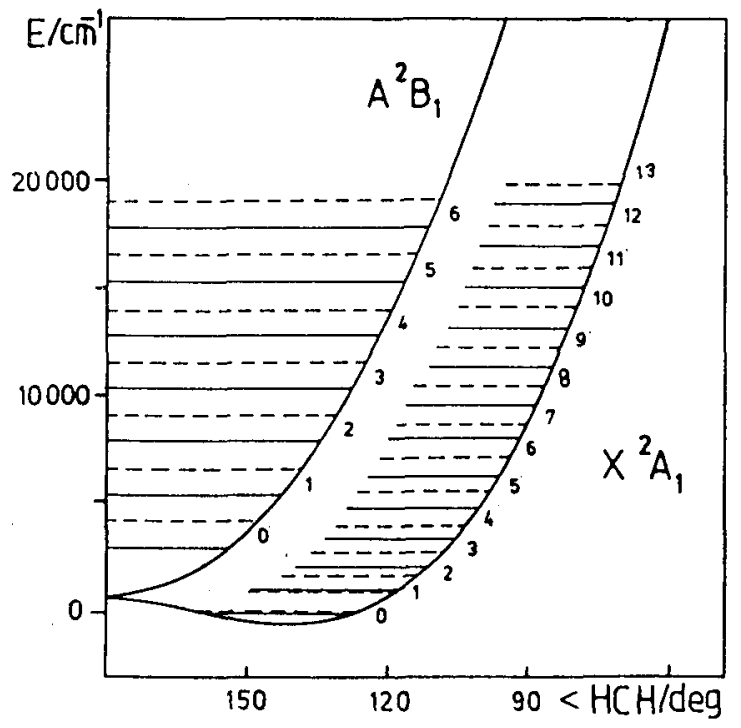

Fig. 5. Calculated potential curves for $\mathrm{CH}_{2}^{+}$and vibrational levels: full lines $K=0$, dashed lines $K=1$ quantum numbers.

The lowest $\mathrm{CH}_{2}^{+}$state is a ${ }^{2} \Pi$ and is split by the Renner-Teller effect into the $\mathrm{X}^{2} \mathrm{~A}_{1}$ state in which the molecule is slightly bent (around $140^{\circ}$ ) and the $\mathrm{A}^{2} \mathrm{~B}_{1}$ state which prefers the linear geometry. The barrier to linearity is predicted to be of the order of $1000-1100 \mathrm{~cm}^{-1}$ (Bartholomae et al. 1981, Reuter \& Peyerimhoff 1992).

In principle the three steps for a purely ab initio treatment of the spectrum are (a) to compute the potential energy surface of the Renner-Teller coupled pair according to Eq.1, (b) to generate the vibrational wavefunctions (considering large-amplitude motion) for the three modes on the two "unperturbed" surfaces and to add rotational functions for motion about the z-axis (linear axis) when solving Eq. 2, and (c) to couple the functions describing nuclear motion on the two surfaces to produce the vibronic functions in a similar manner as shown pictorially for the diatomic molecule $\mathrm{MgO}$ in Figs.3 and 4. Since we found very little coupling between the symmetric stretching and bending modes in $\mathrm{CH}_{2}^{+}$we have explicitly treated only the large-amplitude bending motion in the effective one-dimensional potential which includes the optimized $\mathrm{CH}$ distance at each point (for further discussion see Reuter \& Peyerimhoff 1992). Details of the vibronic mixing will again depend substantially on the relative energetic location 
of the unperturbed levels. The calculated intensity distribution (i.e. the square of the vibronic transition moment which is the quantity in Eq. 3 without the factor $2 / 3$ and the energy) for transitions from the lowest ground state level $v_{2} "=0, K=0$ to $v_{2}^{\prime}=n, K=1$ is plotted in Fig. 6 .

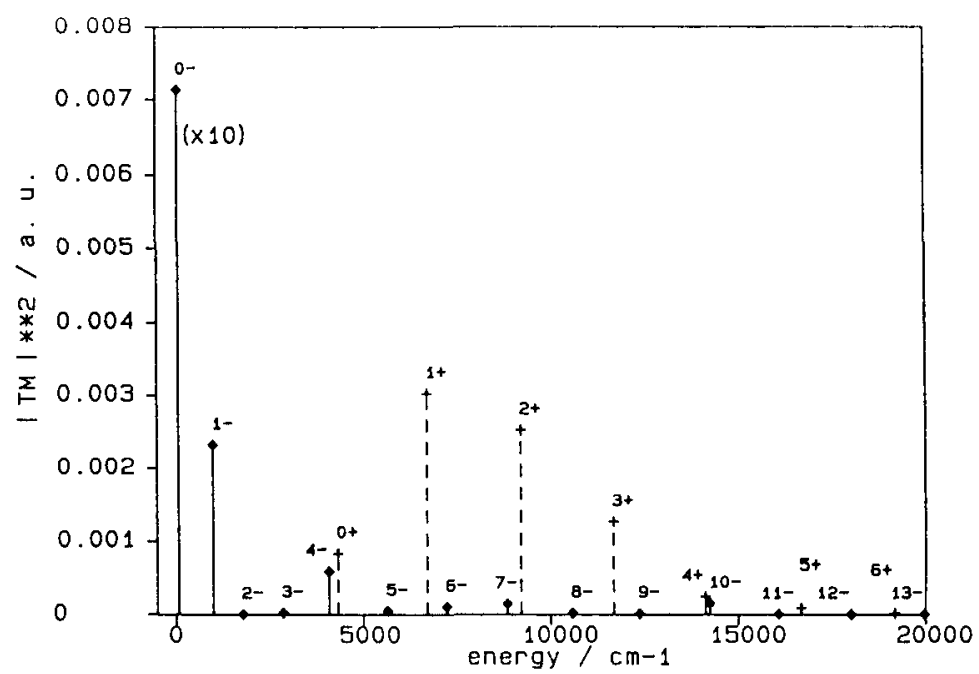

Fig. 6. Calculated intensity distribution (square of vibronic transition moment) from $v^{\prime \prime}=0, K=$ to $v^{\prime}=n, K=1$ levels in $\mathrm{CH}_{2}^{+}$. Levels which have dominant lower-state character are denoted by a minus, those with dominant upper state ${ }^{2} B_{1}$ character by a plus sign.

For low quantumnumbers the typical "infrared" intensity pattern is seen, i.e. high intensity for the $\Delta v=1$ transition, rapidly decreasing for the $\Delta v=2,3$ transitions. The first intensity borrowing is seen for the combination $v^{\prime}=0$ of the upper and $v^{\prime \prime}=4$ of the lower state (levels of essentially upper state character are denoted by a plus, those with dominant lower state character by a minus) so that formally the $v "=0$ to $v "=4$ becomes a much stronger absorber while $v^{\prime \prime}=0$ to $v^{\prime}=0$ is weaker than expected. Similar calculations have been carried out for the other isotopomers $\mathrm{CHD}^{+}, \mathrm{CD}_{2}^{+}$, as well as for transitions from $v "=0$, $K=1$ to $v^{\prime}=n, K=0$ (Reuter \& Peyerimhoff 1992).

Furthermore, this vibronic coupling is apparent in other properties; predictions have been made for the spin-orbit splitting of the vibronic levels (Reuter $\&$ Peyerimhoff 1992) and the vibronically averaged hyperfine coupling constants (Engels \& Peric 1992). All these results can be used as guidelines to interpret and understand the overall structure of the $\mathrm{CH}_{2}^{+}$spectrum; numerically accurate details require higher accuracy in the $\Delta E$ values of the two states than are normally achieved by the computations. Therefore an experiment from which this difference could be determined to wavenumber accuracy would be very helpful for calibration of the potential surfaces which would then allow the accurate prediction of many properties of the $\mathrm{CH}_{2}^{+}$spectrum. 


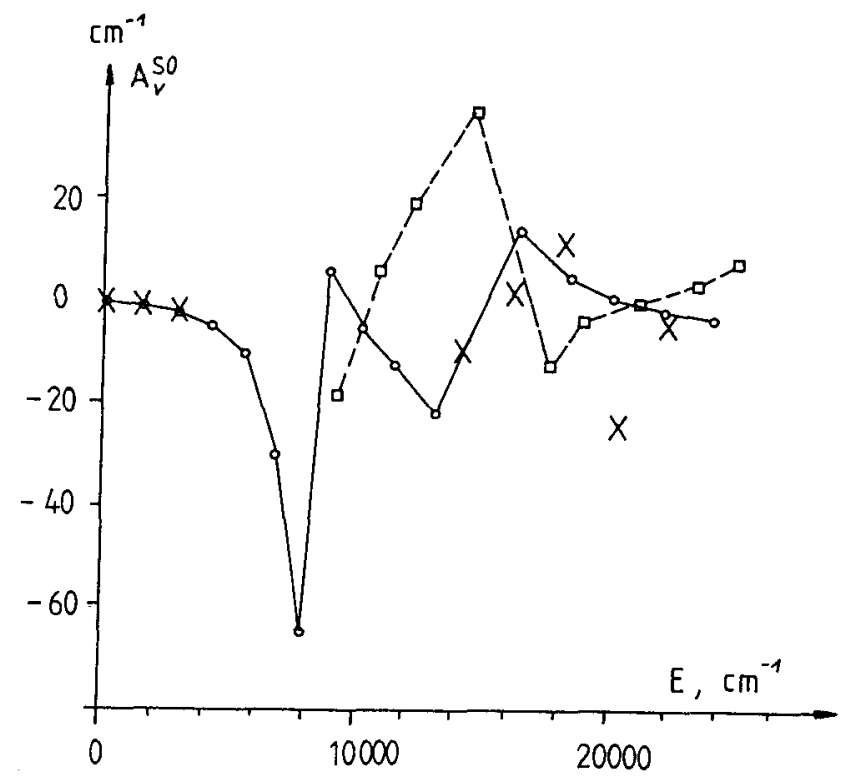

Fig. 7. Calculated spin-orbit splitting of the vibronic levels of $\mathrm{H}_{2} \mathrm{O}^{+}$. The crosses are experimental data. Solid lines connect the vibronic levels of dominant $\mathrm{X}^{2} \mathrm{~A}_{1}$ character, dashed lines those of dominant $\mathrm{A}^{2} \mathrm{~B}_{2}$ character.

A comparison with the $\mathrm{H}_{2} \mathrm{O}^{+}$spectrum, treated in an equivalent manner (Reuter et al. 1991), shows convincingly good correspondence between the measured and the theoretically predicted results. Fig. 7 compares the measured and calculated spin-orbit splitting of the various vibronic levels. As a result of the computational analysis the suggestion made earlier (Weis et al. 1989), namely that the first observed upper state vibronic level should be assigned to $v_{2}^{\prime}=1$ rather than to $v_{2}^{\prime}=0$, is strongly supported. Thus we are confident that the numbering of the ${ }^{2} \mathrm{~A}_{1}$ vibronic levels in earlier analyses (Jungen et al. 1980) should be corrected by one unit. The calculated barrier to linearity is then $8235 \mathrm{~cm}^{-1}$ (Reuter et al. 1991) or $7948 \mathrm{~cm}^{-1}$ (Weis et al. 1989) and not 1000 wavenumbers higher (9187 $\mathrm{cm}^{-1}$ ) as assumed in empirical fits (Jungen et al. 1980).

\section{The spectrum of $\mathrm{C}_{2} \mathrm{H}$}

The spectrum of $\mathrm{C}_{2} \mathrm{H}$ is even more complicated than that of $\mathrm{CH}_{2}^{+}$because (a) it results from a three-state problem and (b) the CC-stretching motion and the bending motion are strongly coupled. An extended ab initio study was performed by Peric et al. (1992).

$\mathrm{C}_{2} \mathrm{H}$ has two low-energy states: ${ }^{2} \Sigma^{+}$and ${ }^{2} \Pi$. At small internuclear CC separations $(1.2 \AA)$ the ${ }^{2} \Sigma^{+}$state lies below the ${ }^{2} \Pi$, around $1.3 \AA$ the two states are almost isoenergetic and at larger separations $(>1.4 \AA)$ the ${ }^{2} \Pi$ state is the 
lowest energy state. In addition, the Renner-Teller effect is operative so that the ${ }^{2} \Pi$ state splits into the two components ${ }^{2} \mathrm{~A}$ ' and ${ }^{2} \mathrm{~A}$ " upon bending. The ${ }^{2} \mathrm{~A}$ ' component interacts (analogously to the situation in Fig. 2) with the ${ }^{2} \Sigma^{+}$state which also takes on ${ }^{2} \mathrm{~A}$ ' symmetry upon bending. As a net result the vibronic wavefunctions possess character from the ${ }^{2} \Sigma^{+}$state and from the two RennerTeller components derived from the ${ }^{2} \Pi$ state (in analogy to the situation in $\mathrm{CH}_{2}^{+}$) with variable mixing coefficients. As expected, the absorption spectrum is very complex. Based on ab initio calculations (Perić et al. 1992) all lines observed to date in ${ }^{12,13} \mathrm{C}$ and ${ }^{1,2} \mathrm{H}$ could be assigned. Many more are predicted so that the experimental search is facilitated. In particular a few "puzzles" have been solved. Examples are:

(a) In several experiments the energy difference between the (011) vibronic level $\left(\mathrm{T}=2091 \mathrm{~cm}^{-1}\right)$ and the $(001)$ level $\left(\mathrm{T}=1841 \mathrm{~cm}^{-1}\right)$ was found to be $250 \mathrm{~cm}^{-1}$. This is significantly different from the $\nu_{2}$ fundamental energy of $372 \mathrm{~cm}^{-1}$. These findings are almost exactly reproduced by the calculations which show an energy difference of $262 \mathrm{~cm}^{-1}\left(\mathrm{~T}=2142 \mathrm{~cm}^{-1}\right.$ and $\mathrm{T}=$ $1880 \mathrm{~cm}^{-1}$ ) and show that the cause for the unexpected (001) and (011) difference is not an unusually large anharmonicity but a level shift due to vibronic mixing of the $\mathrm{X}^{2} \Sigma^{+}$and $\mathrm{A}^{2} \Pi$ states.

(b) The lowest $K=1$ vibronic state shows a measured spin-orbit splitting of $-0.34684 \mathrm{~cm}^{-1}$, unexpected for a ${ }^{2} \Sigma$ state. The computed value for the vibronically coupled ${ }^{2} \Sigma-^{2} \Pi$ level of $-0.35 \mathrm{~cm}^{-1}$ agrees excellently with the measurements.

(c) The measured ratio of the splittings of the lines in the ESR spectra of $\mathrm{C}_{2} \mathrm{H}$ and $\mathrm{C}_{2} \mathrm{D}$ attributed to the hyperfine structure of the hydrogen isotope is 6.33 which differs significantly from the expected value of 6.51 . The calculations predict a value of 6.32 and offer a simple explanation: since the ground state vibronic levels in $\mathrm{C}_{2} \mathrm{D}$ lie somewhat lower than in $\mathrm{C}_{2} \mathrm{H}$ they have a somewhat smaller percentage of the electronic ${ }^{2} \Pi$ character $\left({ }^{2} \Pi\right.$ is the higher state in this case). Since the computed values for the isotropic hyperfine coupling constant at linear geometry are about $45 \mathrm{MHz}$ for the $\Sigma$ state and about -43 $\mathrm{MHz}$ for the $\Pi$ state, the actual percentage of $\Sigma-\Pi$ mixing in the vibronic level is important and the calculations show that vibronically averaged value for the isotropic hfcc should be slightly larger in $\mathrm{C}_{2} \mathrm{D}$ than in $\mathrm{C}_{2} \mathrm{H}$.

\section{Summary}

The present contribution has shown in a few representative examples that modern ab initio calculations are far enough advanced to not only yield potential energy surfaces but also to predict many other quantities of spectroscopic interest with quite high accuracy. Results of this quality require that the electronic wavefunctions be of high accuracy by using at least doublezeta atomic orbital basis sets (see also Langhoff \& Bauschlicher this volume) with additional polarizationtype functions; furthermore, a considerable amount of electron correlation must 
be accounted for in the theoretical treatment employed. For all examples the multireference configuration-interaction method (MRD-CI) was chosen (Buenker et al. 1978, Buenker \& Peyerimhoff 1983). Among the properties discussed (other than energies) the hyperfine coupling seems to be the most sensitive to the CI expansion length, while spin-orbit coupling or electronic transition probabilities seem to show less dependence on the rather large tail (several 100000 compared to first few 10000 configurations) of the CI expansion.

\section{References}

Bruna P.J., Peyerimhoff S.D., 1987, in: Ab initio Methods in Quantum Chemistry I (ed. K. P. Lawley), 1

Herzberg G., 1950, in: Molecular Spectra and Molecular Structure, Vol.1, van Nostrand, 20, 382

Peyerimhoff S.D., 1992, In: E.Kochanski (ed.), Photoprocesses in Transition Metal Complexes, Biosystems and Other Molecules, Kluwer Acad. Publ., 89

Lorenzen-Schmidt H., Perić M., Peyerimhoff S.D., 1993, J. Chem. Phys., 98, 525

Perić M., Peyerimhoff S.D., 1993, J. Chem. Phys., 98, 3587

Perić M., Peyerimhoff S.D., 1993, J. Mol. Structure, in press

van Hemert M., Dohmann H., Peyerimhoff S.D., 1986, Chem. Phys., 110, 55

van Hemert M., Peyerimhoff S.D., 1991, J. Chem. Phys., 94, 4369

Thümmel H., Klotz R., Peyerimhoff S.D., 1989, Chem. Phys., 135, 229

Gemein B., Peyerimhoff S.D., 1991, Chem. Phys. Letters, 184, 45

Marian C.M., 1990, J. Chem. Phys., 93, 1176

Hogness T.R., Kvalness H.M., 1928, Phys. Rev., 32, 942

Herzberg G., 1961, Can. J. Phys., 39, 1511

Rösslein M., Gabrys C.M., Jagod M.-F., Oka T., 1992, J. Mol. Spectry, 153, 738

Bartholomae R., Martin D., Sutcliffe B.T., 1981, J. Mol. Spectry, 87, 367

Carter S., Handy N.C., 1984, Mol. Phys., 52, 1367

Sutcliffe B.T., Tennyson J., 1987, J. Chem. Soc. Faraday Trans.II, 83, 1663

Lee J.S., Secrest D., 1988, J. Phys. Chem., 92, 1821

Reuter W., Peyerimhoff S.D., 1992, Chem. Phys., 160, 11

Engels B., Perić M., 1992, J. Chem. Phys., 97, 7629

Reuter W., Perić M., Peyerimhoff S.D., 1991, Mol. Phys., 74, 569

Weis B., Carter S., Rosmus P., Werner H.-J., Knowles P.J., 1989, J. Chem. Phys., 91, 2818

Jungen Ch., Hallin K.-E., Merer A.J., 1980a, Mol. Phys., 40, 25

Jungen Ch., Hallin K.-E., Merer A.J., 1980b, Mol. Phys., 40, 65

Perić M., Peyerimhoff S.D., Buenker R.J., 1992, Z. Phys. D., 24, 177

Buenker R.J., Peyerimhoff S.D., Butscher W., 1978, Mol. Phys., 35, 771

Buenker R.J., Peyerimhoff S.D., 1983, in: New Horizons of Quantum Chemistry, (eds. P. O. Löwdin, B. Pullmann), 183 